069

\section{CASE SERIES OF ATRIAL FLUTTER WITH 1:1 CONDUCTION: A COMPARATIVE ANALYSIS AMONG GROUPS OF PATIENTS SUFFERING SPONTANEOUS VERSUS DRUG INDUCED 1:1 CONDUCTING ATRIAL FLUTTER}

V N Nathwani, K W Wong, M J Jones, C H Hayes, K R Rajappan, T B Betts, Y B Bashir Oxford University Hospitals

doi:10.1136/heartjnl-2013-304019.69

Background Atrial flutter with 1:1 (AFL 1:1) conduction may be spontaneous or secondary to anti-arrhythmic drug (AAD) use. This group of patients are not well characterised. We aimed to compare various characteristics between patients suffering 'spontaneous' AFL 1:1 (the development of 1:1 Atrioventricular conducting Atrial Flutter in the absence of concomitant $\mathrm{AAD}$ use) and 'Drug-induced' AFL 1:1.

Methods This is a retrospective study of patients referred to our institution over an 8 year period. Sixty-eight patients had confirmed and documented atrial flutter with 1:1 conduction.

Results Baseline clinical characteristics and differences between the study populations are summarised in Table.

Table 1

\begin{tabular}{lllll}
\hline & Overall & Spontaneous & Drug-induced & p Value \\
\hline Age & $62 \pm 14$ & $62 \pm 14$ & $61 \pm 13$ & 0.86 \\
Male (\%) & 79 & 79 & 80 & 1.0 \\
Structural heart disease (\%) & 36 & 40 & 33 & 0.63 \\
LV impairment (\%) & 11 & 16 & 3 & 0.13 \\
LA diameter (mm) & $4.2 \pm 0.7$ & $4.3 \pm 0.8$ & $4.0 \pm 0.4$ & 0.14 \\
CHADS VASc (Median) & 1 & 1 & 1 & 1.0 \\
Flutter rate (bpm) & $218 \pm 38$ & $251 \pm 32$ & $212 \pm 33$ & $<0.0001$ \\
Broad complex & 64 & 62 & 70 & 0.58 \\
tachycardia (\%) & & & & $<0.0001$ \\
Concomitant AF (\%) & 49 & 28 & 80 & \\
\hline
\end{tabular}

Patients with spontaneous AFL 1:1 had a significantly higher flutter rate and were less likely to have concomitant AF. Drug-induced AFL 1:1 was mainly secondary to Class 1 AAD's despite the use of AV blocking agents in a third of these patients. Sixty-three patients underwent a successful cavotricuspid isthmus ablation with no recurrence.

Conclusions We have reported the largest series of patients with atrial flutter and 1:1 conduction. Patients suffering spontaneous AFL 1:1 had a significantly faster flutter rate compared to the drug-induced population. 